\title{
Prevalência de sífilis em gestantes de um município mineiro no período de 2015-2020
}

\author{
Prevalence of syphilis in pregnant women from a city in Minas Gerais, Brazil, in the period \\ 2015-2020 \\ Prevalencia de sífilis en gestantes de una ciudad de Minas Gerais, Brasil, en el período \\ 2015-2020
}

Karolayne Sabrina de Morais ${ }^{1 *}$, Arthur Henrique Resende Porto ${ }^{1}$, Débora da Penha Herculano ${ }^{1}$, Luana Matos Silva Araújo ${ }^{1}$, Jaquelina Elvira Marques de Oliveira², Tânia Maria Delfraro Carmo, Talane Alcântara de Oliveira ${ }^{3}$, Policardo Gonçalves da Silva1.

\section{RESUMO}

Objetivo: Descrever e analisar a prevalência do diagnóstico de sífilis em gestantes, bem como seus determinantes sociais, no município de Passos/MG no período de janeiro de 2015 a junho de 2020. Métodos: Trata-se de pesquisa descritiva, retrospectiva, com abordagem quantitativa e qualitativa, realizada a partir de dados do Sistema de Informação de Agravos de Notificação (SINAN). Foram incluídas como participantes do estudo pacientes que tenham realizado acompanhamento junto ao Programa Materno-Infantil (PROMAI) da Santa Casa de Misericórdia de Passos (SCMP). Foi executada, ainda, estratificação segundo raça, faixa etária, trimestre gestacional de diagnóstico e tratamento concomitante do parceiro. Resultados: 121 casos de sífilis gestacional (SG) foram atendidos pelo PROMAI no período em questão, sendo 89 provenientes de Passos e 32 de outros municípios da região. Foi percebido, em sua maioria, um perfil de gestantes jovens e com diagnóstico tardio, sem correlação de raça. A informação acerca do tratamento concomitante dos parceiros foi, em sua maioria, desconhecida. Conclusão: Acreditase que esse estudo possa contribuir com a temática bem como evidenciar a necessidade de capacitações profissionais voltadas para o preenchimento adequado das fichas de notificação do SINAN, objetivando reduzir subnotificações e identificar perfis fidedignos das pacientes.

Palavras-chave: Sífilis, Gravidez, Prevalência.

\begin{abstract}
Objective: To describe and analyze the prevalence of syphilis diagnosis in pregnant women, as well as their social determinants, in the town of Passos/MG from January 2015 to June 2020. Methods: This was a descriptive, retrospective research, with a quantitative and qualitative approach, carried out based on data from the Notifiable Diseases Information System (SINAN). The study participants included patients who had been followed up by the Maternal and Child Program (PROMAI) of Santa Casa de Misericórdia de Passos (SCMP). Stratification was also performed according to race, age group, gestational trimester of diagnosis and concomitant treatment of the partner. Results: 121 cases of gestational syphilis (GS) were attended by PROMAI in the period in question, 89 from Passos and 32 from other municipalities in the region. Mostly, a profile of young pregnant women with a late diagnosis was found, with no correlation of race. The information about the concomitant treatment of the partners was, for the most part, unknown. Conclusion: We believe that this study can contribute to the theme as well as highlight the need for professional training aimed at properly filling out the SINAN notification forms, aiming to reduce underreporting and identify reliable patient profiles.
\end{abstract}

Key words: Syphilis, Pregnancy, Prevalence.

\footnotetext{
${ }^{1}$ Universidade do Estado de Minas Gerais (UEMG), Passos - MG.

*E-mail: karolayne.2118573@discente.uemg.br

2 Escola Técnica Dr. Adail Nunes da Silva (CEETEPS), Taquaritinga - SP.

${ }^{3}$ Coordenação Estadual de IST, Aids e Hepatites Virais de Minas Gerais, Belo Horizonte - MG.
} 


\section{RESUMEN}

Objetivo: Describir y analizar la prevalencia del diagnóstico de sífilis en gestantes, así como sus determinantes sociales, en el municipio de Passos / MG desde enero de 2015 a junio de 2020. Métodos: Se trata de un estudio transversal, conducido con 161 individuos, de ambos sexos, edad de 20 a 59 años. Se trata de una investigación descriptiva, retrospectiva, con enfoque cuantitativo y cualitativo, realizada con base en datos del Sistema de Información de Enfermedades Notificables (SINAN). Los participantes del estudio incluyeron pacientes que habían sido seguidos por el Programa Materno Infantil (PROMAI) de la Santa Casa de Misericórdia de Passos (SCMP). También se realizó la estratificación según raza, edad, trimestre gestacional de diagnóstico y tratamiento concomitante de la pareja. Resultados: 121 casos de sífilis gestacional (SG) fueron atendidos por PROMAI durante el período en cuestión, 89 de Passos y 32 de otros municipios de la región. Se notó, en su mayoría, un perfil de gestantes jóvenes y con diagnóstico tardío, sin correlación racial. La información sobre el tratamiento concomitante de las parejas era, en su mayor parte, desconocida. Conclusión: Se cree que este estudio puede contribuir a la temática, así como resaltar la necesidad de una formación profesional orientada a rellenar adecuadamente los formularios de notificación del SINAN, con el objetivo de reducir el subregistro e identificar perfiles confiables de pacientes.

Palabras clave: Sífilis, Embarazo, Prevalencia.

\section{INTRODUÇÃO}

A sífilis permanece como um grave problema de saúde pública no mundo. No Brasil, apesar de todos os recursos disponíveis para sua extinção, como métodos diagnósticos de baixo custo e tratamento efetivo desde a década de 1950, sua incidência continua em crescimento. Trata-se de uma Infecção Sexualmente Transmissível (IST), que tem como agente etiológico a bactéria espiroqueta Treponema palidum e cujo risco de transmissão está diretamente relacionado ao contato com as lesões mucocutâneas provocadas pela doença, mais comuns no primeiro ano de infecção (BRASIL, 2019b).

A sífilis gestacional (SG) é a forma da doença desenvolvida por uma mulher durante a gravidez que, quando não tratada adequadamente, pode culminar na propagação do patógeno para o feto, a chamada transmissão vertical (TV). Essa forma de transmissão pode se dar tanto no ambiente intraútero como durante a passagem do feto pelo canal do parto, sendo a probabilidade da ocorrência de sífilis congênita influenciada pelo estágio da sífilis na mãe e pela duração da exposição fetal. Toda gestante com sífilis deve ser estratificada como alto risco por necessitar de consultas mais frequentes (MOREIRA KFA, et al., 2017; SOARES LG, et al., 2017).

Pesquisas mostram que, na América Latina, a cobertura do diagnóstico da sífilis nas gestantes que realizaram o pré-natal no ano de 2014 foi de apenas $61 \%$. Já no Brasil, surgem, a cada ano, cerca de 937 mil novos casos de sífilis, sendo a prevalência em gestantes de 2,6\%, o que corresponde a quase 50 mil gestantes com sífilis e aproximadamente 12 mil casos de sífilis congênita (NONATO SM, et al., 2015; BRASIL, 2019a).

O grande responsável pela coleta e pelo processamento dos dados sobre as notificações em todo o território nacional é o Sistema de Informação de Agravos de Notificação (SINAN). No entanto, os dados coletados não refletem a magnitude total da infecção, dado que 0 preenchimento das fichas pelos profissionais de saúde responsáveis nem sempre são adequados segundo os protocolos nacionais. Nesse sentido, o grande número de informações "ignoradas" ou em branco pode comprometer o estabelecimento de um perfil epidemiológico de qualidade acerca da sífilis no Brasil (NETO LG, et al., 2019).

A atuação do enfermeiro na atenção primária à saúde (APS) é essencial no combate à SG, pois essa é a principal porta de entrada dos usuários ao Sistema Único de Saúde (SUS), possibilitando um elo próximo entre profissional e paciente. Além disso, os enfermeiros são os agentes que abastecem as bases de dados do SINAN através do preenchimento das fichas de notificação, sendo sua atividade crucial para o mapeamento dos pontos críticos do sistema. Por fim, juntamente com os demais membros da equipe podem aproveitar a ocasião do pré-natal para a realização de atividades educativas, enfatizando a importância de medidas de prevenção, realização dos testes rápidos durante o acompanhamento pré-natal 
e orientações para a paciente no momento do possível diagnóstico (CAVALCANTE PAM, et al., 2017; SHUBERT CO, et al., 2018; SILVA PTB, et al., 2019).

Com relação à assistência pré-natal, é recomendado pelo Ministério da Saúde (MS) que as gestantes realizem o exame de Venereal Disease Research Laboratory (VDRL) em dois momentos: na primeira consulta e na 30aㅗ semana de gestação. Além disso, devem repeti-lo na ocasião do parto, a fim de assegurar tratamento precoce ao recém-nascido, em caso de não tratamento ou de reinfecção da gestante. São consideradas tratadas de forma correta as gestantes que tenham recebido dose adequada de penicilina $\mathrm{G}$ benzatina, com conclusão do esquema 30 dias previamente ao parto e tratamento concomitante do parceiro sexual (BRASIL, 2019b; LOPES HH e MANDUCA AVG, 2018).

Observa-se que, no Brasil, têm-se publicado muitos estudos sobre a SG. Entretanto, poucos deles avaliam os seus potenciais determinantes. Ademais, mesmo a existência de alta cobertura de pré-natal às gestantes, a oferta de técnicas diagnósticas rápidas e de baixo custo e a disponibilidade de tratamento efetivo não têm sido suficientes até os dias de hoje para a sua erradicação (PADOVANI C, et al., 2018).

Outro importante desafio que persiste é o tratamento concomitante dos parceiros sexuais dessas gestantes, que devem, de acordo com protocolos estabelecidos pelo MS, receber o mesmo esquema terapêutico de suas companheiras. Isso guarda relação intrínseca com o sucesso do tratamento pois, além de impedir a reinfecção da mulher e a consequente transmissão para o feto, a adesão do parceiro aumenta as chances da mulher de concluir a terapêutica, devido ao apoio recebido. Contudo, grande parte desses parceiros ainda não são tratados, o que se deve tanto à desinformação, reflexo de baixa escolaridade e ausência de medidas educativas voltadas para esses indivíduos, como a uma abordagem inadequada e/ou insuficiente pelas equipes de saúde (VASCONCELOS MIO, et al., 2016).

Nesse contexto, a presente pesquisa é de extrema importância, fundamentando-se na necessidade de demonstração do perfil epidemiológico das pacientes notificadas com SG, nas implicações desse diagnóstico e na necessidade de aprimorar o atendimento a essa população. Assim, o objetivo geral do estudo é descrever e analisar a prevalência do diagnóstico de sífilis, bem como seus determinantes sociais, em um município mineiro, no período de janeiro de 2015 a junho de 2020.

\section{MÉTODOS}

O estudo consiste em uma pesquisa descritiva, retrospectiva, com abordagem quantitativa e qualitativa, realizada a partir de fontes secundárias (dados do Sistema de Informação de Agravos de Notificação (SINAN)) acerca da prevalência de sífilis em gestantes no município de Passos/MG.

O município consta com 114.458 habitantes e se estende por 1338,1 quilômetros quadrados, sendo localizado na região sul de Minas Gerais (IBGE, 2019). A cidade conta com 24 Unidades Básicas de Saúde, compondo a Atenção Primária; com a Unidade de Pronto Atendimento (UPA) e o Ambulatório Escola da Universidade do Estado de Minas Gerais (Ambes-UEMG) como principais representantes da Atenção Secundária e com 2 serviços de nível terciário, que são a Santa Casa de Misericórdia de Passos (SCMP) e o Hospital São José.

Foram incluídas como participantes do estudo mulheres gestantes com sorologia positiva para sífilis, cujas notificações tenham sido feitas no período de janeiro de 2015 a junho de 2020 e que tenham realizado acompanhamento junto ao Programa Materno-Infantil (PROMAI), serviço multidisciplinar da SCMP, com ações voltadas para o atendimento integral da mulher e do binômio mãe-filho.

A coleta de dados foi realizada por meio do SINAN, utilizando-se uma tabulação dos dados epidemiológicos da população em questão. Foi executada, também, estratificação segundo os critérios de raça, faixa etária, trimestre gestacional em que ocorreu o diagnóstico e tratamento concomitante do parceiro sexual. Em seguida, as informações foram organizadas usando-se o software Microsoft Excel. Por fim, os dados quantitativos foram analisados mediante técnica de estatística descritiva com o auxílio da ferramenta de análise Visual Basic for Applications (VBA), disponível no software supracitado. Os resultados foram, então, expressos em forma de figuras e tabelas. 
Previamente à execução do trabalho, foi encaminhada carta de solicitação de autorização ao serviço de vigilância epidemiológica do município de Passos, requerendo acesso aos dados disponíveis no SINAN. Posteriormente à autorização, foi agendada a visita dos pesquisadores ao referido departamento para a execução da coleta. Uma vez que foi utilizada apenas a tecnologia disponível em um sítio de domínio público, sem coleta de informações relativas aos sujeitos ou descrições sobre as situações assistenciais, não houve necessidade de submeter o trabalho a um comitê de ética em pesquisa.

\section{RESULTADOS}

Foram notificados, no período de 2015 a 2020, 121 casos de sífilis gestacional (SG) dentre mulheres atendidas pelo Programa Materno-Infantil da Santa Casa de Misericórdia de Passos (PROMAI), sendo 89 casos provenientes do município de Passos e 32 de outros municípios da região. Esses dados foram coletados por meio do Sistema de Informação de Agravos de Notificação (SINAN).

A Tabela 1 mostra a quantidade de casos notificados, por ano, oriundos dos demais municípios da região. As cidades foram: Alpinópolis, Bom Jesus da Penha, Capetinga, Carmo do Rio Claro, Cássia, Delfinópolis, Fortaleza de Minas, Guapé, Itaú de Minas, Nova Resende, Pratápolis, São João Batista do Gloria, São José da Barra e São Roque de Minas. Para essas pacientes não foi realizada estratificação por categorias, como foi feito para as pacientes residentes de Passos, uma vez que o estudo tinha como foco apenas esse município.

Tabela 1 - Distribuição de casos de sífilis em gestante nos últimos cinco anos, que por algum motivo consultou no Programa Materno-Infantil da Santa Casa de Misericórdia de Passos. Passos, MG, Brasil, 2015-2020.

\begin{tabular}{lcc}
\hline Ano & Cidade & Número de Casos \\
\hline 2015 & Capetinga & 1 \\
2015 & Cássia & 1 \\
2015 & Pratápolis & 3 \\
2015 & São João Batista do Glória & 1 \\
2016 & Alpinópolis & 1 \\
2016 & Bom Jesus da Penha & 1 \\
2016 & Itaú de Minas & 3 \\
2016 & São José da Barra & 1 \\
2017 & Alpinópolis & 1 \\
2017 & Bom Jesus da Penha & 1 \\
2017 & Delfinópolis & 1 \\
2017 & Itaú de Minas & 2 \\
2017 & São José da Barra & 1 \\
2018 & Carmo do Rio Claro & 1 \\
2018 & Guapé & 2 \\
2018 & Itaú de Minas & 2 \\
2019 & Cássia & 2 \\
2019 & Fortaleza de Minas & 1 \\
2019 & Itaú de Minas & 1 \\
2019 & Nova Resende & 1 \\
2020 & Cássia & 1 \\
\hline
\end{tabular}

Fonte: Morais KS, et al., 2021; dados extraídos do SINAN, 2020.

O Gráfico 1 por sua vez, mostra a frequência, por ano, das notificações provenientes do município de Passos-MG. Destaca-se que, em 2018, houve a descentralização dos testes rápidos para a Atenção Primária à Saúde (APS), o que justifica um aumento notável no número de casos notificados, tendo em vista que o acesso à testagem rápida foi facilitado, observando-se uma diferença de 9 casos a mais em relação ao ano anterior. Já no ano de 2020, houve um menor número de casos, tendo em vista que as notificações foram apuradas somente até o mês de junho. 
Gráfico 1 - Frequência de casos de sífilis em gestante segundo ano de notificação. Passos, MG, Brasil, 2015-2020.

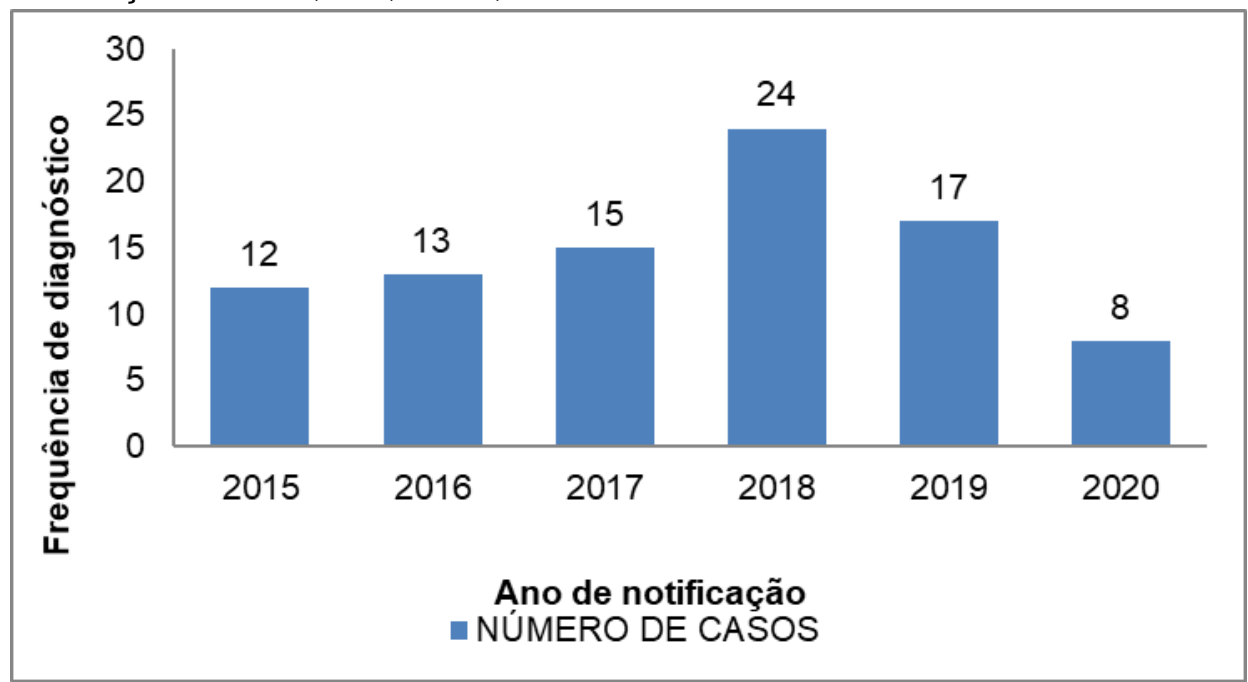

Fonte: Morais KS, et al., 2021; dados extraídos do SINAN, 2020.

Para as gestantes residentes de Passos foi realizada estratificação do perfil segundo os seguintes critérios: raça, faixa etária, momento da gravidez em que ocorreu o diagnóstico e tratamento concomitante dos parceiros. O Gráfico 2 por exemplo, mostra a frequência por raça, revelando que a maior prevalência de casos ocorreu em mulheres pardas, sendo 37 ao todo. Já mulheres brancas representaram um total de 34 notificações e mulheres pretas 18 .

Gráfico 2 - Frequência de casos de sífilis em gestante segundo raça. Passos, MG, Brasil, 2015-2020.

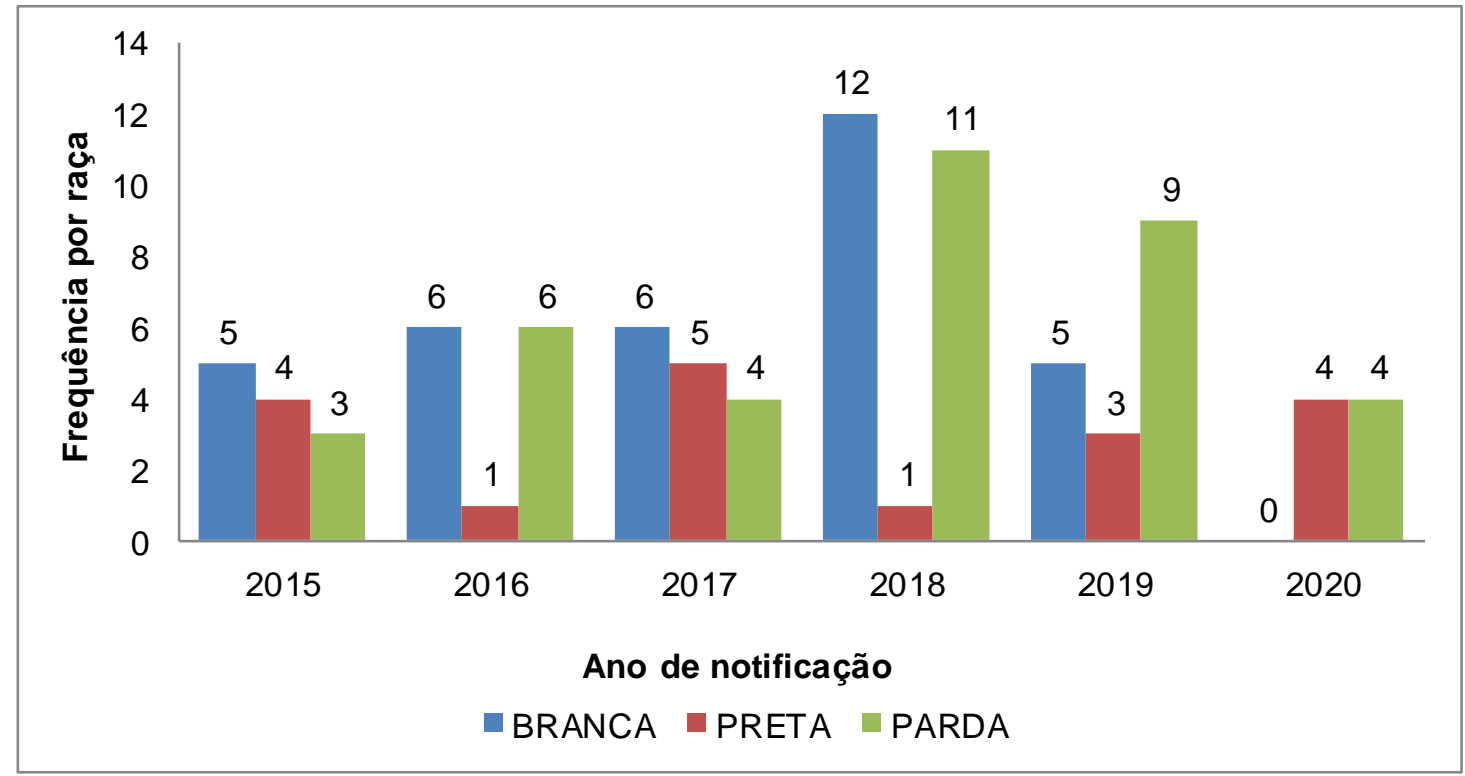

Fonte: Morais KS, et al., 2021; dados extraídos do SINAN, 2020.

Os resultados apresentados no Gráfico 3, em relação à idade das pacientes, mostram que houve maior número de casos notificados na faixa etária dos 20 aos 34 anos gerando, assim, grande preocupação pelo fato de mulheres jovens serem as mais afetadas. Soma-se a isso o fato de os casos na faixa etária dos 15 aos 19 anos totalizarem 19, nos levando a acreditar em um cenário em que ainda há casos de novas infecções subnotificadas, deixando evidente a necessidade de se intensificar a abrangência e a qualidade 
do rastreamento durante o pré-natal. Por fim, um caso foi relatado na faixa dos 10 aos 14 anos e 8 casos na faixa dos 35 aos 49 .

Gráfico 3 - Frequência de casos de sífilis em gestantes segundo faixa etária. Passos, Minas Gerais, Brasil, 2015-2020.

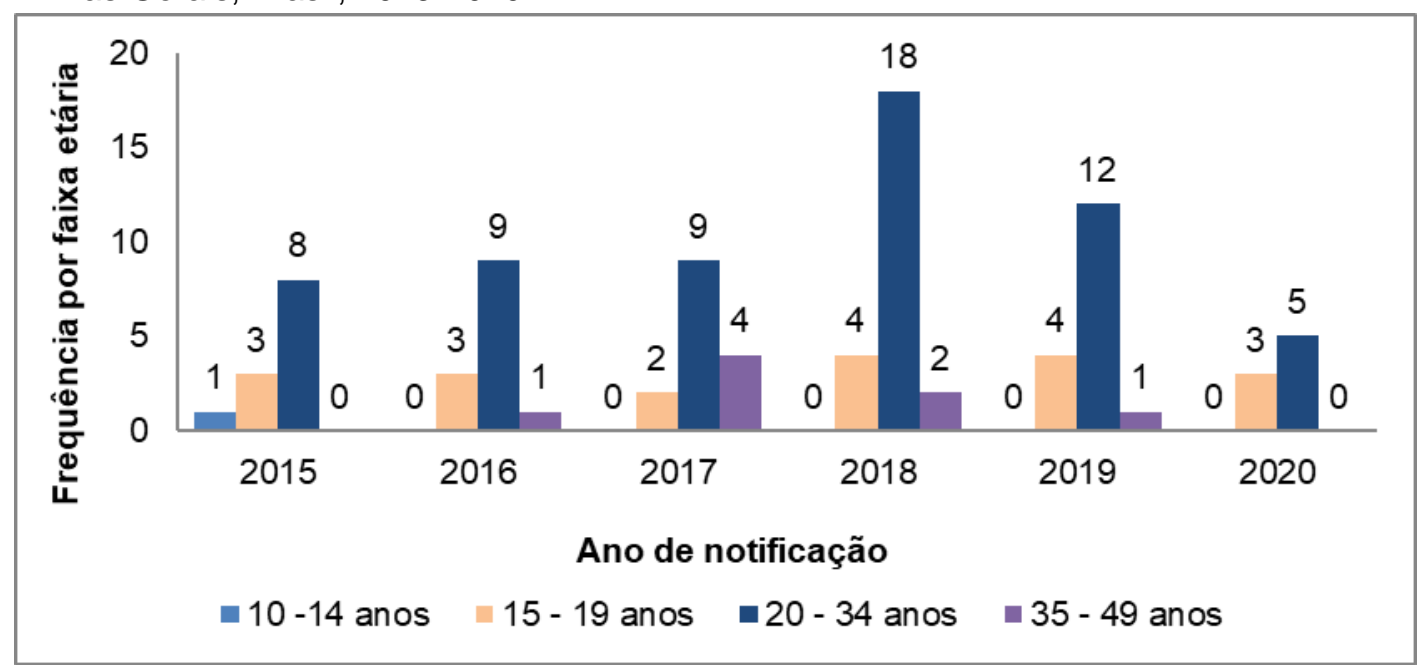

Fonte: Morais KS, et al., 2021; dados extraídos do SINAN, 2020.

A frequência de diagnóstico por trimestre de gestação, descrita no Gráfico 4, mostra que um grande número de notificações realizadas no quarto trimestre (27 notificações), sendo esse último o período dos 3 primeiros meses após o parto. Esse dado traz grande preocupação, uma vez que se trata de diagnósticos feitos após o período gestacional, o que representa, possivelmente, um grande número de casos de sífilis congênita, o que seria facilmente evitável por meio de acompanhamento pré-natal. Sobre casos diagnosticados durante a gestação em si, foram 19 no primeiro trimestre, 22 no segundo e 21 no terceiro.

Gráfico 4 - Frequência de casos de sífilis em gestantes segundo momento de diagnóstico. Passos, MG, Brasil, 2015-2020.

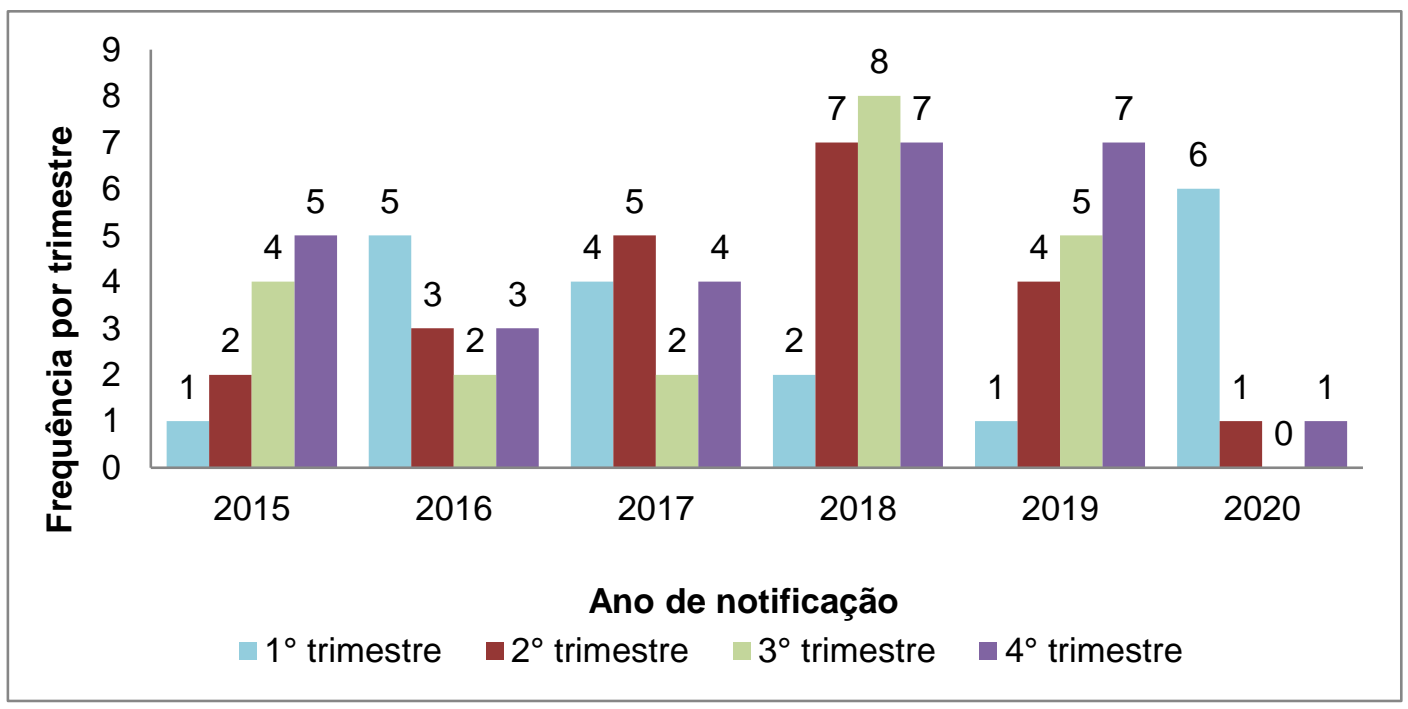

Fonte: Morais KS, et al., 2021; dados extraídos do SINAN, 2020.

Por fim, o Gráfico 5 realiza a estratificação de acordo com o tratamento dos parceiros. Destaca-se que em 2015, para todos os casos notificados, esse dado foi registrado como "ignorado" enquanto, nos anos subsequentes, para muitos casos essa informação também era desconhecida. Esse fato deixa claro que, infelizmente, o tratamento das parcerias sexuais é um aspecto pouco valorizado no diagnóstico, nos 
levando a acreditar em um cenário muito além do conhecido em relação aos casos de sífilis e suas diversas manifestações.

Gráfico 5 - Frequência de casos de sífilis em gestantes segundo tratamento concomitante do parceiro. Passos, MG, Brasil, 2015-2020.

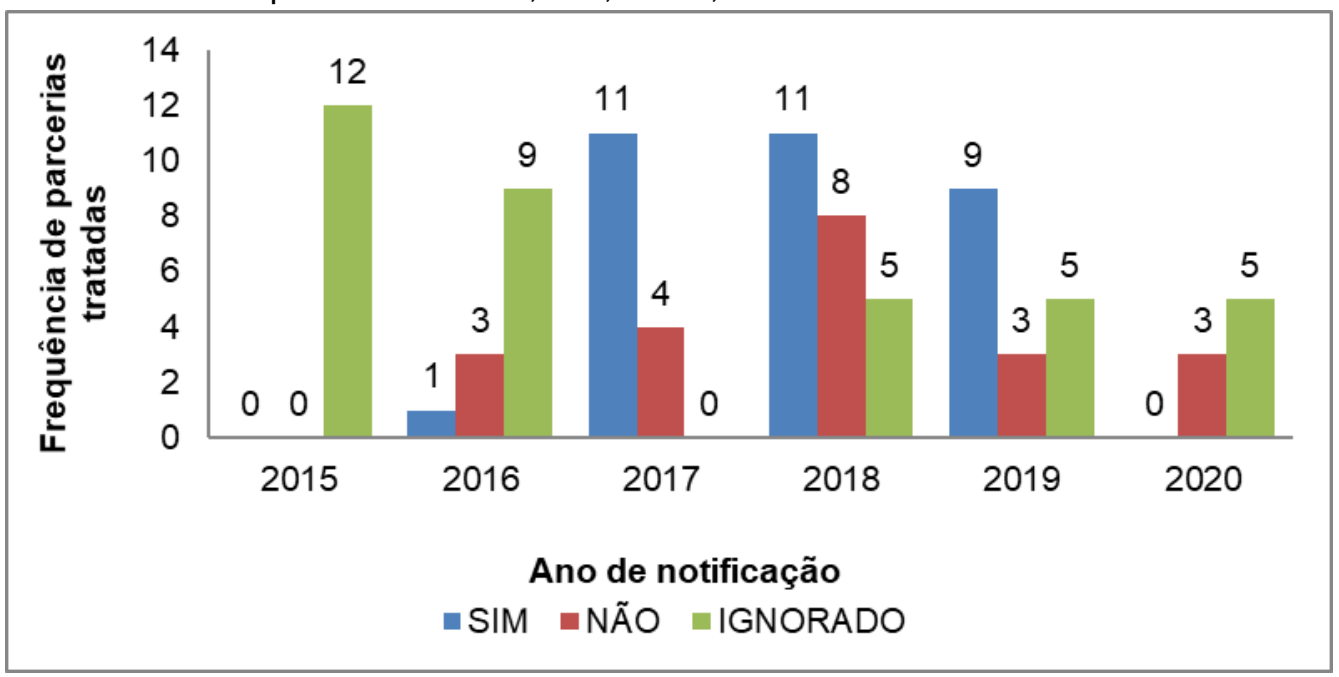

Fonte: Morais KS, et al., 2021; dados extraídos do SINAN, 2020.

\section{DISCUSSÃO}

A Organização Mundial da Saúde (OMS) apresenta como meta a eliminação da sífilis congênita, definida como ocorrência de 0,5 ou menos casos de sífilis congênita para cada mil nascidos vivos, sendo essa meta também adotada pelo Ministério da Saúde do Brasil (DOMINGUES RMSM e LEAL MC, 2016).

Todavia, há anos a sífilis vem crescendo no Brasil, mesmo com medidas capazes de conter esse avanço. Observou-se nesse trabalho, por exemplo, que, nos anos de 2015 e 2016, o número de casos foi pequeno em relação aos anos subsequentes. Além disso, a maior parte das mulheres foi diagnosticada no $4^{\circ}$ trimestre, ou seja, após o parto. Esses indicadores refletem a baixa qualidade do pré-natal no país e a pouca importância que os profissionais de saúde (gestores ou diretamente envolvidos no atendimento) têm dado ao diagnóstico e ao tratamento da sífilis.

Por sua vez, dados mais recentes do boletim epidemiológico do Ministério da Saúde ressaltam que, entre os anos de 2017 e 2018, a sífilis adquirida teve um aumento de $32,7 \%$ e, a SG, 20,9\%. Cabe destacar, nesse sentido, que se trata de uma infecção fortemente relacionada à vulnerabilidade social, sendo a caracterização das regiões de maior ocorrência fundamental para auxiliar os profissionais de saúde e gestores no planejamento de ações (BRASIL, 2019a; CONCEIÇÃO HN, et al., 2019).

Em outubro de 2018, o governo recomendou medidas de combate à doença, dentre as quais o incentivo à realização do pré-natal (ainda no primeiro trimestre da gestação, com o intuito de identificar precocemente e diminuir a transmissão vertical), a ampliação do diagnóstico por meio de testes rápidos e o tratamento oportuno para a gestante e seu parceiro com 0 uso de penicilina benzatina, considerada 0 medicamento mais seguro e eficaz para esses casos. Contudo, um estudo conduzido entre 2012 e 2013 mostrou que menos de um quarto das gestantes realizou exame físico apropriado e pouco mais da metade receberam todas as orientações preconizadas, revelando carência significativa nessa área (SANTANA MVS, et al., 2019; SILVA PG, et al., 2021).

O aumento na incidência da infecção pode ser atribuído a diversos fatores, dentre os quais o crescimento das notificações e a ampliação da cobertura de testagem associada à difusão dos testes rápidos diagnósticos. Isso se deu principalmente com o alargamento de cobertura da Estratégia de Saúde 
da Família (ESF), associada à distribuição desses métodos de testagem para as Unidades Básicas, além de ações de vigilância epidemiológica direcionadas para uma melhor abordagem e identificação dos casos da doença (CAMARA LS, et al., 2020).

Com relação ao fator étnico, apesar de algumas pesquisas apontarem que a sífilis está associada às mulheres pardas e pretas, nesse estudo a maioria foi identificada como branca ou parda. Contudo, como não houve diferença significativa entre os grupos, não foi possível identificar associação entre a prevalência de sífilis e a cor da pele. Por outro lado, um fator fortemente associado à sífilis materna é a faixa etária das mulheres, fato corroborado pelos resultados: entre as 89 gestantes com sorologia positiva para sífilis, $68,53 \%$ possuem entre 20 e 34 anos. Pode-se deduzir a partir disso que mulheres mais jovens e solteiras possuem vidas mais ativas e geralmente possuem maior grau de liberdade sexual, se envolvendo com mais de um parceiro e por vezes não utilizando preservativo. Assim, ficam mais vulneráveis a contraírem infecções (TENÓRIO LV, et al., 2020).

Com relação ao momento de diagnóstico da sífilis nas gestantes, a maioria se deu no terceiro e no quarto trimestres. Nesse sentido, os desfechos da não identificação e tratamento precoces da infecção durante a gestação podem ser graves para o bebê, incluindo eventos como prematuridade, aborto, natimortalidade e óbito neonatal. Esses danos são diretamente dependentes da fase da infecção materna e da idade gestacional da exposição fetal. Por conseguinte, uma assistência pré-natal de qualidade, com captação precoce da gestante (de preferência no primeiro trimestre) com ações de promoção da saúde, orientação sexual e reprodutiva e realização do protocolo dos exames preconizados durante o período gestacional, é essencial para a prevenção de danos ao bebê e também da mulher (PADOVANI C, et al., 2018).

Ademais, grande parte dos infectados pela sífilis não têm conhecimento de tal fato, devido à escassez de sintomas nos estágios iniciais da doença, podendo transmiti-la ao parceiro. Nesse tocante, o presente estudo observou-se que $23,6 \%$ dos parceiros não foram tratados, nos levando a acreditar em circunstâncias como ausência de contato ou distanciamento para com a gestante, sorologia não reagente e/ou recusa ao tratamento. Em contrapartida, na maioria das fichas preenchidas no serviço hospitalar não constavam informações a esse respeito, favorecendo o indício de subnotificação para registro no SINAN, o que dificulta o planejamento de ações no controle da doença.

Destarte, o tratamento adequado do parceiro sexual para sífilis representa um dos principais desafios no controle da sífilis congênita, tendo em vista a falta de política específica para esta situação e as dificuldades inerentes à relação da gestante e seu parceiro. A não realização de tratamento do parceiro sexual da gestante com sífilis impede a quebra da cadeia de transmissão da doença e pode invalidar todas as medidas de controle instituídas durante o cuidado pré-natal, mesmo no caso das gestantes tratadas adequadamente e em tempo oportuno (NUNES IR, et al., 2020; VILELA LSCAL, et al., 2019).

Assim, é importante não perder oportunidades para se realizar as ações de prevenção da transmissão vertical. Diante de qualquer sinal ou sintoma clínico e/ou sorologia não treponêmica positiva, e na impossibilidade de confirmação do diagnóstico, a conduta é tratar imediatamente a gestante e orientá-la a notificar seu parceiro para que realize o tratamento conjunto, evitando, assim a reinfecção da mulher. $O$ tratamento deve ser realizado na própria unidade onde foi realizado o diagnóstico, não necessitando de internamento hospitalar (CABRAL BTV, et al., 2017).

Destaca-se, também, o papel da educação em saúde na prevenção da SG. O apoio dos profissionais de saúde (principalmente dos enfermeiros), aos pacientes é essencial para uma correta orientação, bem como para o esclarecimento de possíveis dúvidas que venham a surgir durante o atendimento oferecido. Percebese, no entanto, que há ainda uma grande falha nesse processo, evidenciando a necessidade de fortalecimento dos currículos de graduação acerca do tema e de um constante processo de educação continuada, que deve ocorrer mesmo após a formação dos profissionais. A abordagem deles para com os pacientes deve acontecer não apenas no sentido de mera transmissão passiva de conhecimento, mas possuir caráter coletivo e empoderador, de modo a garantir a participação ativa dos usuários no cuidado de sua própria saúde (NOBRE CS, et al., 2018). 
Diante do que foi exposto, vale ressaltar a necessidade de fortalecimento das ações de integração intersetoriais, que possam fortalecer a descentralização da testagem rápida para todas as unidades básicas de saúde, bem como a realização do tratamento imediato, tanto pelas gestantes como por suas parcerias. Além disso, ações de sensibilização dos profissionais no seguimento dos protocolos vigentes, bem como o estímulo à implantação e fortalecimento dos Comitês de Investigação da Transmissão Vertical das IST são de extrema importância no controle e redução desse agravo (SILVA PG, et al., 2021).

\section{CONCLUSÃO}

A pesquisa identificou um aumento da prevalência de sífilis entre gestantes no município de Passos, o que parece estar acontecendo também em outras regiões do país. Esse aumento pode estar relacionado ao maior acesso a diagnóstico, mas também ser decorrente do crescimento de práticas sexuais desprotegidas. Foi percebido um perfil de gestantes, em sua maioria, jovens e com diagnóstico tardio, sem correlação de raça. O tratamento concomitante dos parceiros foi, em grande parte dos casos, desconhecido. Acredita-se que esse estudo possa contribuir com a temática e evidenciar a necessidade de capacitações profissionais voltadas para o preenchimento adequado das fichas de notificação do SINAN, objetivando reduzir subnotificações e identificar um perfil mais fidedigno das pacientes.

\section{REFERÊNCIAS}

1. BRASIL. Ministério da Saúde. Boletim Epidemiológico Sífilis. 2019a.

2. BRASIL. Ministério da Saúde. Protocolo Clínico e Diretrizes Terapêuticas para a prevenção da transmissão vertical de HIV, Sífilis e Hepatites Virais. 2019b.

3. CABRAL BTV, et al. Sifílis em gestante e sífilis congênita: um estudo retrospectivo. Revista Ciência Plural, 2017; 3(3): 32-44.

4. CAMARA LS, et al. Perfil epidemiológico das gestantes com sífilis no Rio de Janeiro, Revista Research, Society and Development, 2020; 9(10): e4999108712.

5. CAVALCANTE PAM, et al. Sífilis gestacional e congênita em Palmas, Tocantins, 2007-2014. Revista Epidemiologia e Serviços de Saúde, 2017; 26(2): 255 - 264.

6. CONCEIÇÃO HN, et al. Análise epidemiológica e espacial dos casos de sífilis gestacional e congênita. Revista Saúde em Debate, 2019; 43(123): 1145-1158.

7. DOMINGUES RMSM, LEAL MC. Incidência de sífilis congênita e fatores associados à transmissão vertical da sífilis: dados do estudo Nascer no Brasil. Revista Cadernos de Saúde Pública, 2016; 32(6): e00082415.

8. LOPES HH, MANDUCA AVG. Diagnóstico e tratamento da sífilis na gestação. Revista de Patologia do Tocantis, 2018; 5(1): 58-61

9. MOREIRA KFA, et al. Perfil dos casos notificados de sífilis congênita. Revista Cogitare Enfermagem, 2017; 22(2), 1-10.

10. NETO LG, et al. Epidemiologia da sífilis gestacional e congênita no estado de Goiás no período de 2013 a 2018. Revista Brasileira Militar de Ciências, 2019; 5(13): 64-71.

11. NOBRE CS, et al. Sistema de saúde no controle da sífilis na perspectiva das enfermeiras. Revista Enfermagem UERJ, 2018; 26: e12527

12. NONATO SM, et al. Sífilis na gestação e fatores associados à sífilis congênita. Revista Epidemiologia e Serviços de Saúde, 2015; 24(4), 681-694.

13. NUNES IR, et al. Sífilis congênita: caracterização epidemiológica no estado do Piauí, Brasil. Revista Eletrônica Acervo Saúde, 2020; Sup(50): e755.

14. PADOVANI C, et al. Sífilis na gestação: associação das características maternas e perinatais em região sul do Brasil. Revista Latino-americana de Enfermagem, 2018; 26(1): e3019.

15. SANTANA MVS, et al. Sífilis Gestacional na Atenção Primária. Revista Diversitas Journal, 2019; 4(2), 403-419

16. SHUBERT CO, et al. Transmissão vertical da sífilis: o enfermeiro e as ações de prevenção. Revista Ciência Atual, 2018; 11(1): 2-13

17. SILVA PTB, et al. A assistência do profissional enfermeiro frente ao diagnóstico da sífilis no período gestacional: uma revisão bibliográfica. Revista Terra \& Cultura, 2019; 35(esp): 78-92.

18. SOARES LG, et al. Sífilis gestacional e congênita: características maternas e neonatais. Revista Brasileira de Saúde Materno Infantil, 2017; 17(4): 781-789.

19. TENÓRIO LV, et al. Fatores que dificultam 0 diagnóstico precoce da sífilis na gestação. Revista Research, society and development, 2020; 9(9): e377997225.

20. SILVA PG, et al. Produção e validação de tecnologia educacional sobre cuidados de enfermagem para prevenção da sífilis. Revista Brasileira de Enfermagem, 2021; 74(5): e20190694.

21. VASCONCELOS MIO, et al. Sífilis na gestação: estratégias e desafios dos enfermeiros da atenção básica para o tratamento simultâneo do casal. Revista Brasileira em Promoção da Saúde, 2016; 29(Supl): 85-92

22. VILELA LSCAL, et al. O pré-natal como ferramenta na prevenção da sífilis congênita: uma revisão integrativa da literatura. Revista Brazilian Journal of Health Review, 2019; 2(3): 1609-1615. 